\title{
Solitary Fibrous Tumour of Tentorium: A Case Report
}

\author{
${ }^{1}$ Dr N.L.N.Moorthy ${ }^{, 2}$ Dr S. Padmaja,${ }^{3}$ Dr M.Vithaleshwar Rao,${ }^{4} \mathrm{Dr}$ \\ B.G.Ratnam \\ ${ }^{1}$ professor Radiology, ${ }^{2}$ senior Resident Radiology, ${ }^{3}$ assistant Professor Radiology, ${ }^{4}$ neurosurgeon \\ Department Of Radiology, Apollo Institute Of Medical Science And Research \\ Hyderabad
}

\begin{abstract}
Solitary fibrous tumours are rare mesenchymal soft tissue tumours arising from multiple sites in the body including the dura and the intracranial ventricles. These masses are grouped along with rare haemangiopericytomas which form about less than $0.4 \%$ of all CNS tumours. Locally aggressive solitary fibrous tumours closely mimic other dural based tumours most likely meningiomas. We report a case of biopsy proven solitary fibrous tumour from tentorium operated in a 60 year old adult and discuss the imaging features in other related dural based masses.
\end{abstract}

\section{Introduction}

Many neoplastic and non neoplastic lesions involve the dura, of which meningioma is the most common dural based tumour. The others include haemangiopericytoma, lymphoma, dural metastases, RosaiDorfman disease, solitary fibrous tumour, Ebstein-Barr virus associated smooth muscle tumours, melanocytic neoplasms, Erdheim-Chester disease, sarcoidosis, gliosarcoma, tuberculosis etc. Solitary fibrous tumor belongs to a group of mesenchymal spindle cell masses that arise mostly from pleura, extrathoracic soft tissues, and meninges. These tumours are both benign and malignant. Haemangiopericytoma is considered as the aggressive form of solitary fibrous tumour by many authors ${ }^{1}$. Clinically presenting similar to meningioma, occur mostly along the tentorium, falx, cerebellopontine angle and in the spinal dura. On CT, solitary fibrous tumour appears as well circumscribed hyperattenuating extraaxial dural based masses with underlying smooth bone erosion . On MR imaging these tumours have T2 heterogenous signal intensity and show intense contrast enhancement. Flow voids are common within the tumour. MR Spectroscopy shows lipid, lactate and myo-inositol peaks. Dural tail sign is rare. Angiography shows profound tumour blush .Histopathology showed neoplastic tissue composed of sheets of cells with oval to spindled nuclei and scanty cytoplasm with scattered mitotic figures (4$5 / 10$ hpf). There was no necrosis. Immuno his to chemistry report was IHC-794-17, neoplastic cells are positive.

\section{Case report}

A 60 year old male presented with progressive head ache in occipital region since one year with associated hearing loss in left ear, facial numbness, imbalance, dysarthria and urinary incontinence. Clinical examination revealed ataxic gait, pendular nystagmus with bilateral lateral and up external gaze. No history of seizures, or loss of consciousness or vomiting. Routine lab investigations were normal. Contrast enhanced MRI brain showed a well defined large non homogenously enhancing extraaxial mass measuring $6.1 \times 3.8 \times 5.3 \mathrm{cms}$ arising from tentorium on left side. The mass extended on either side of tentorium. On T1 weighted image the mass was mildly hyperintense with hypointensity on T2 weighted images. The mass also showed small areas of cystic changes and blooming on Gradient sequence. There was significant compression of left cerebellum, brain stem and fourth ventricle with resultant moderate degree hydrocephalus and downward herniation of cerebellar tonsils. The lower cranial nerves were seen separately. The supratentorial extension of the mass caused significant compression of occipital and temporal lobes. Enhancing dural tail also noted along the tentorium. MR spectroscopy showed elevated choline, reduced creatine and NAA levels. Alanine absent. A provisional diagnosis of a large extraaxial mass from tentorium was made on MR imaging. Supra and infratentorial craniotomy was done and soft, moderately vascular tumour arising from tentorium with both supra and infra tentorial components was excised and the tentorium was coagulated. Histopathology revealed neoplastic tissue composed of sheets of cells with oval to spindled nuclei and scanty cytoplasm with scattered mitotic figures ( 4-5/10hpf). There was no necrosis.

Immunohistochemistry report: IHC-794-17 .Neoplastic cells are positive for CD 34 and negative for EMA. MIB-I index is $8-10 \%$. With the above findings a diagnosis of solitary fibrous tumour/ hemangiopericytoma grade 2 was made. 


\section{Discussion}

The most common mass lesion involving the dura is meningioma. However many other dural based lesions are described which have similar imaging features of meningioma like solitary fibrous tumour, hemangiopericytoma, primary dural lymphoma, Rosai-Dorfman disease, metastases etc ${ }^{\mathbf{2 , 3}}$.

Hemangiopericytoma: A rare tumour arising from the pericytes surrounding the capillaries occur in younger age than meningioma. It is considered to be part of spectrum of solitary fibrous tumour with locally aggressive form. These tumours cause erosion of underlying bone unlike meningioma. On MR these tumours are typically isointense on both T1 and T2 weighted imaging with prominent flow voids, heterogenous contrast enhancement. Unlike meningioma, hemangiopericytoma have a narrow base of attachment and result in mushrooming into adjacent brain ${ }^{\mathbf{1}}$. MR spectroscopy the tumour show a high myoinositol peak at $3.56 \mathrm{ppm}$ and a lack of alanine whereas meningioma shows low myoinositol with alanine peak ${ }^{4}$. Liu et al ${ }^{\mathbf{5}}$ found minimum apparent diffusion coefficient (Min ADC) is high in hemagiopericytoma than in meningioma. The presence of intratumoral calcification and dural

tail sign are uncommon in hemangiopericytoma. These specific findings in these tumours are well described by many authors in the literature ${ }^{6,7,8,9,10}$. In view of abnormally high vascularity, onyx embolization by direct trancranial puncture of the tumour was performed by Dale Ding and others ${ }^{\mathbf{1 1}}$ and the total resection of the tumour was possible.

Solitary fibrous tumour: belongs to a group of rare mesenchymal spindle cell tumours seen in mostly adults. The common sites involved are meninges ,pleura and soft tissues. These tumours are either benign and malignant. According to WHO classification of tumours of CNS , solitary fibrous tumour/ hemagiopericytoma are grouped with mesenchymal non meningothelial tumours under 3 grades as both SFT and hemangiopericytomas are considered as one entity ${ }^{\mathbf{1 2}}$. In the CNS the sites of involvement include tentorium, falx, occipital and spinal dura, and cp angle ${ }^{\mathbf{1 3}}$. On imaging these tumours are well defined dural

based extra axial masses which are hyperattenuating on CT . Focal calcification may be seen. These tumours erode underlying bone unlike meningiomas. On T2 weighted images the tumour shows heterogenous signals with focal areas of contrast enhancement. A yin-yang appearance is described on T2 weighted imaging. Flow voids are also seen. Dural tail may be seen in some cases. MR spectroscopy shows elevated myo-inositol and lipid lactate peaks unlike meningioma which show elevated alanine. On diffusion weighted imaging the lesion shows restriction ${ }^{14}$. Y.C.Weon ${ }^{15}$ and others reviewed the imaging features in 6 cases of solitary fibrous tumour and found that black and white mixed pattern on T2 weighted imaging may be useful in the diagnosis.

Primary dural lymphoma: seen in middle aged females. The lesions can be single or multiple and hyperattenuating on CT scan. On MR they are hypointense on T2 weighted imaging and show profuse contrast enhancement. Perilesional vasogenic edema is typical. Indistinct brain- tumour interface is suggestive of primary dural lymphoma than menigioma.Metastases: breast, prostate and lungs are common primary sites that produce dural metastases. On imaging dural metastases appear as focal or diffuse dural thickening with associated bone erosion and enhancement with contrast.Rosai-Dorfman disease: is a rare histiocytosis with massive lymphadenopathy with associated CNS involvement. On imaging the lesions are hyeprattenuating on $\mathrm{CT}$ and isointense on T1 weighted and hypointense on T2 weighted imaging unlike meningioma. The lesions enhance with contrast and show dural tail frequently.Epstein-Barr virus associated smooth muscle tumors: ( leiomyoma, leiomyosarcoma ) on CT the lesions are isodense and are dural based. On MR they are hypointense on $\mathrm{T} 1$ weighted and hyperintense on $\mathrm{T} 2$ weighted imaging.

Melanocytic neoplasms: typically appear as hyperattenuating on CT with avid contrast enhancement On MR the lesions are hyperintense on T1 and iso to hypointense on T2 weighted imaging.Erdheim-Chester disease : is a rare histiocytosis, can be single or multiple dural based lesions. The lesions are iso to hypointense on T1 and T2 weighted imaging with enhancement on contrast injection.

Sarcoidosis : these dural based lesions appear as diffuse thickening which are isointense on T1 and hypointense on T2 weighted imaging.In the present case the dural based tumour was mildly hyperintense on T1 w and non homogenously hypointense on T2 weighted imaging and show heterogenous enhancement with contrast administration.The dural tail sign was positive.No intratumoral calcification was found .MR spectroscopy showed elevated choline, reduced creatine, NAA levels with absent alanine. There was no underlying bone erosion.Histopathology with immunohistochemistry confirmed the diagnosis of solitary fibrous tumour. The treatment protocols include preoperative embolization followed by surgery and adjuvant radiotherapy .

\section{Conclusion}

Besides meningioma, other dural based tumours like solitary fibrous tumour has to be considered in the differential diagnosis of these lesions in the brain. 


\section{Legends :}

Figure $(\mathrm{A}-\mathrm{E})$

A) Axial T1weighted B) axial T2 weighted post contrast C) axial , D) coronal E) sagittal MR images show a well defined lobulated mildly hyperintense T1 and hypointense T2 extra axial mass from tentorium with heterogenous contrast enhancement and with significant brain compression.

\section{References}

[1]. Alice Boyd Smith ,Iren Horkanyne-Szakaly, Janson W.Schroeder.Elisabeth J.Rushing Mass lesions of the dura: beyond meningioma-Radiologic-pathologic correlation Radiographics 2014 ; 34: 295-312.

[2]. Mahlon D. Johnson, Suzanne Z. Powell ,Philip J.Boyer, Robert J.Weil, Paul L Moots Dural lesions mimicking meningiomas Hum Pathol 2002 33: 1211-1226.

[3]. Danai Chourmouzi, StamatiaPotsi, Anestis Moumtzouoglou,Elisavet Papadopoulou,Kostas Drevelegas, Thomas Zaraboukas,Antonios Drevelegas Dural lesions mimicking meningiomas ; a pictoral essay World J Radiol 2012 mar 28 ; 4 (3) : 7582.

[4]. Barba I, Moreno A, Martinez-Perez I, et al Magnetic resonance spectroscopy of brain hemangiopericytoma: high myoinositol

[5]. concentrations and discrimination from meningioma J Neurosurg $2001 ; 94(1): 55-60$

[6]. Liu G, Chen ZY, Ma L, Lou X, Li SJ, Wang YL. Intracranial hemangiopericytoma: MR imaging findings and diagnostic usefulness of minimum ADC values. J Magn Reson Imaging $2013 ; 38(5)$ : 1146-1151

[7]. M V Chiechi, J G Smirniotopoulos and H Mena Intra cranial hemangiopericytomas; MR and CT features American journal of neuroradiology, August 1996, 17(7) 1365-1371

[8]. Wei Wu, Ji-xin Sh,Hui-lin Cheng, Han-dong Wang, Chun-hua Hang, Qun-Li, Shi, Hong-xia Yin. Hemangiopericytomas in the central nervous system. Journal of clinical neurosciences April 2009 volume 16, issue 4, pages 519-523.

[9]. N. Mama, A. Ben Abdallah, I.Hasni, K.Kadri, N.Arifa,M.Ladib, K.Till-Graiess MR imaging of hemangiopericytomas. Journal of neuroradiology, December 2014, volume 41, n5 pages 296-306.

[10]. Cong Ma, Feng Xu, Yu-Dong, Xiao, Ramchandra Paudel, Yi Sun, En-Hua Xiao Magnetic resonance imaging of intracranial hemangiopericytoma and correlation with pathological findings. Oncology Letters November 2014, volume 8, issue 5, pages 21402144.

[11]. Haopeng Pang, Zhenwei Yao, Yan Ren, Guobing Liu, Jiawen Zhang, and Xiaoyuan Feng. Morphological patterns and imaging features of intracranial hemangiopericytomas: a retrospective analysis. Onco Targets and Therapy 2015; 8; 2169-2178.

[12]. Dale Ding, Derek Kreitel, and Kenneth C. Liu Onyx Embolisation of an Intracranial Hemangiopericytoma by Direct Transcrania

[13]. Puncture. Interventional Neuroradiology 2013 Dec; 19(4): 466-470.

[14]. Louis D.N.,Perry A,Reitenberger G, et al The 2016 World Health Organization classification of tumors of central nervous systema summary Acta Neuropathol ( june 2016 ) vol 131 issue 6 page 803-820

[15]. 13. Nicolas Penel, Eric Yaovi Amela, Gauthier Decanter, Yves-Marie Robin, and Perrrine Marec-Berard. Review article: Solitary Fibrous Tumors and so-called Hemangiopericytoma Hindawi / Sarcoma volume 2012(2012), Article ID 690251, 6 pages.

[16]. 14. Clarençon F, Bonneville F, Rousseau A, et al Intracranial solitary fibrous tumor: imaging findings Eur J Radiol 2011; 80(2):387-394.

[17]. Y.C.Weon, E.Y.Kim, H.S.Byun, K.Park and J.H.Kim Intracranial Solitary Fibrous Tumors: Imaging Findings in 6 Consecutive Patients. American Journal of Neuroradiology September 2007,28 (8) 1466-1469.

Figure A)

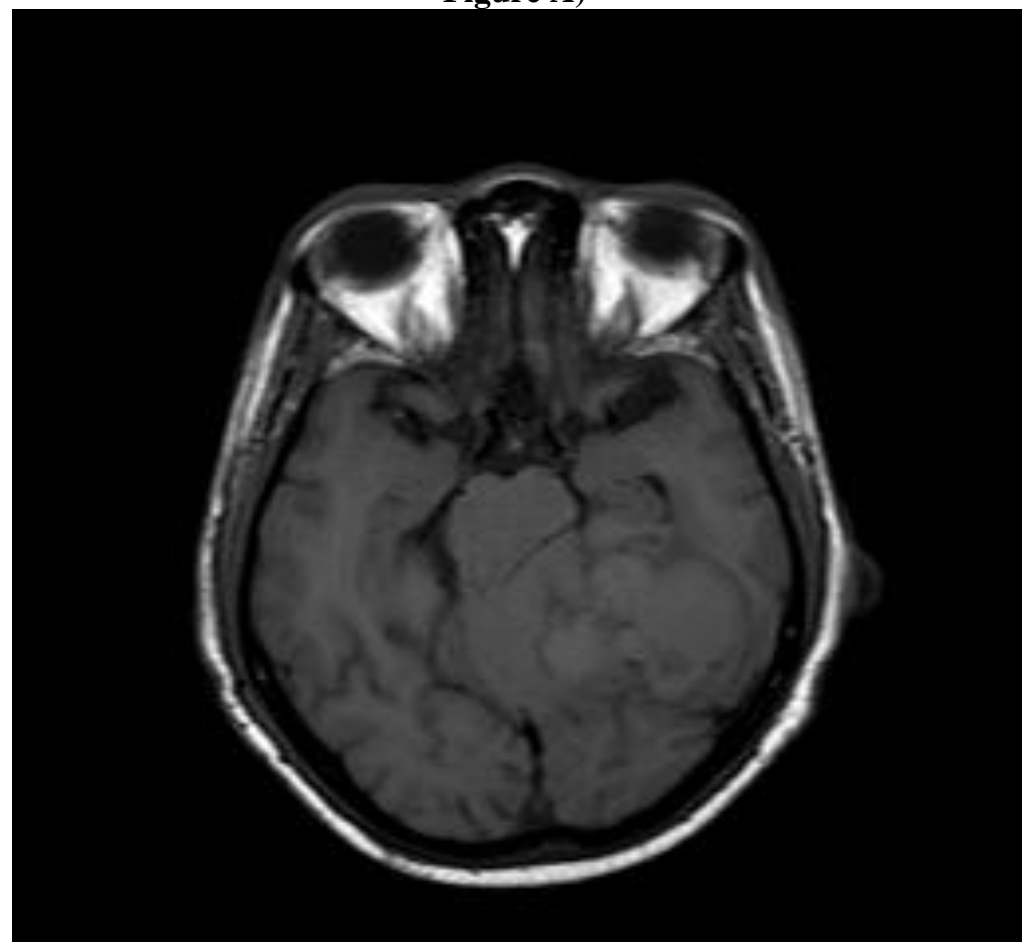


Figure B)

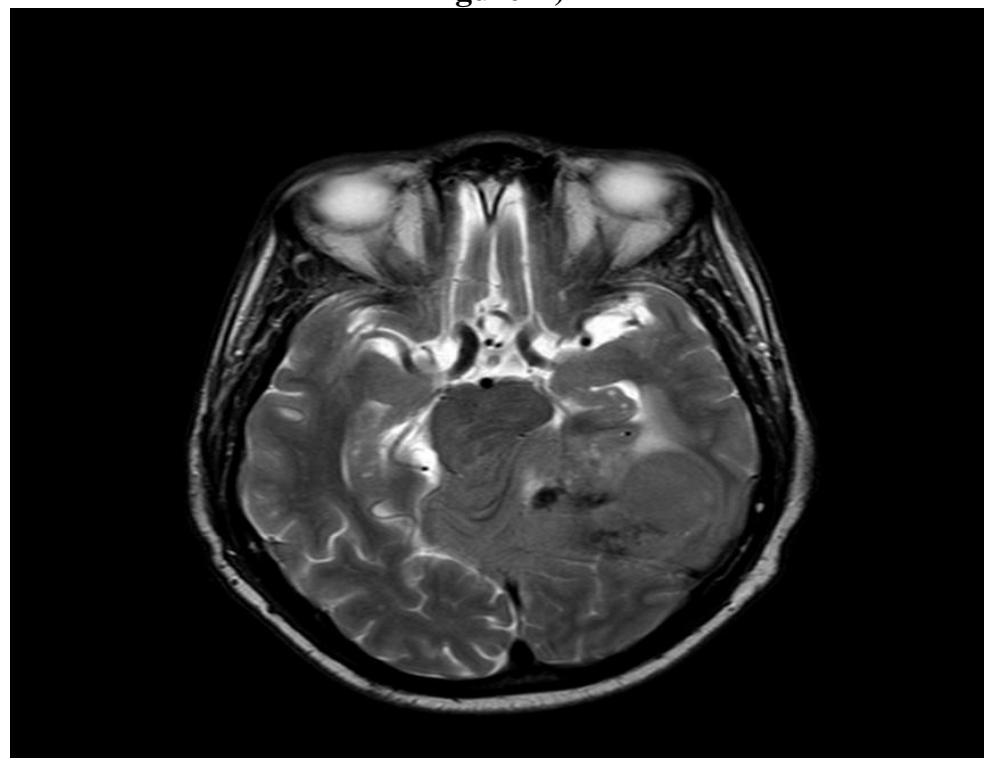

Figure C)

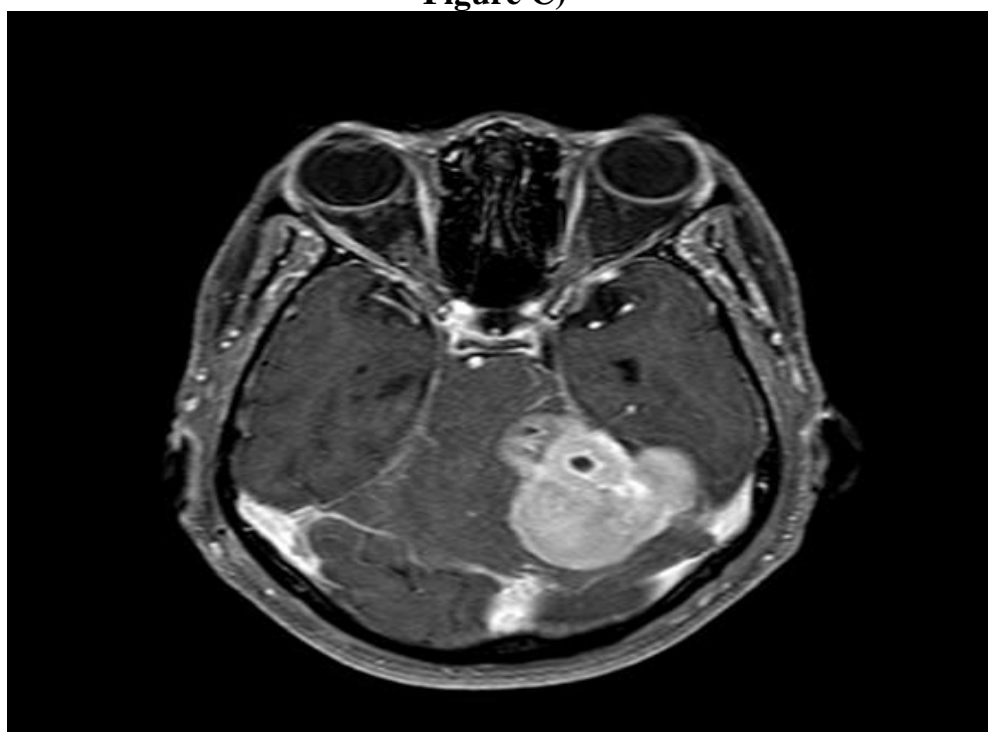

Figure D )

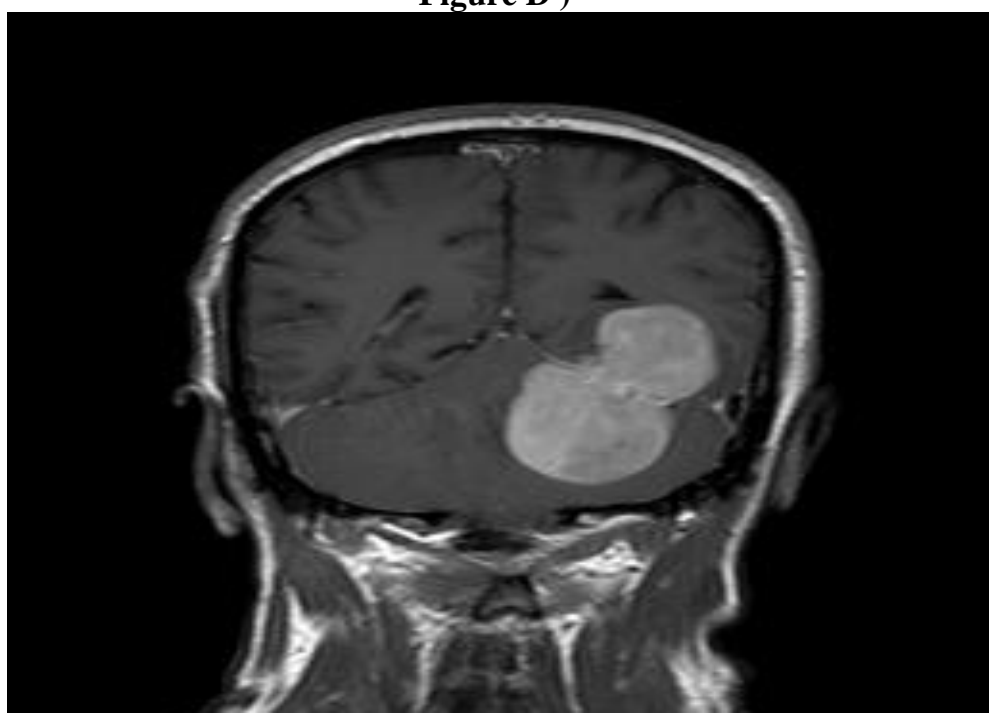




\section{Figure E)}

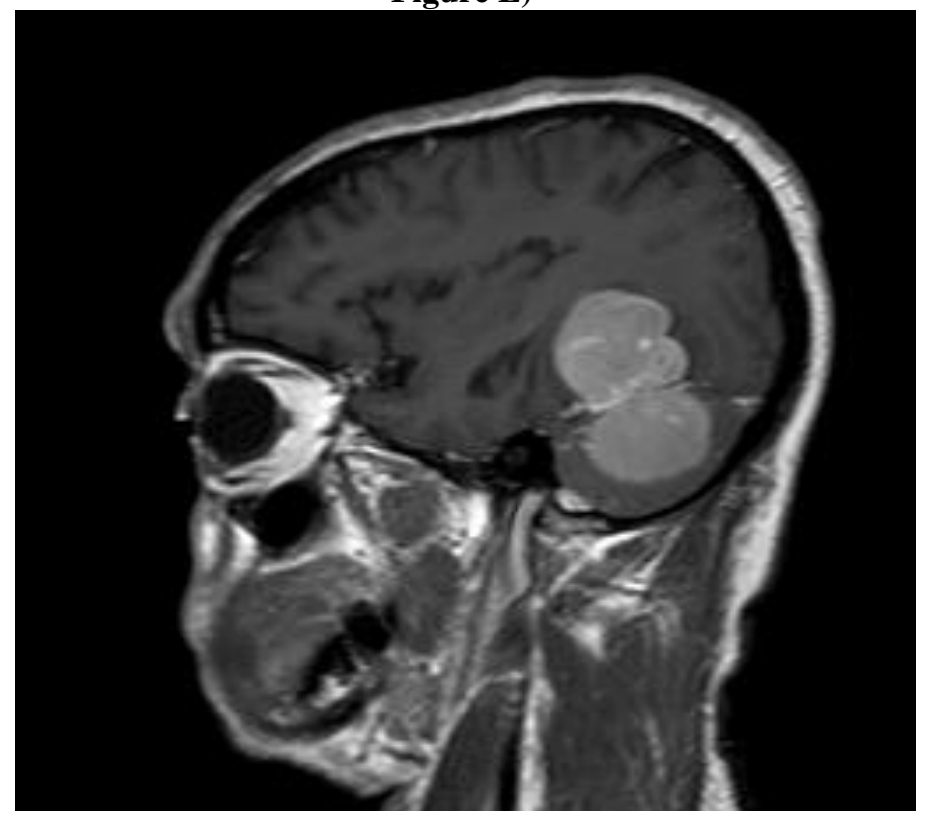

